\title{
USO DA IMAGEM DIGITAL PARA QUANTIFICAÇÃO DE MISTURAS DE AZEITE DE OLIVA E ÓLEO DE CANOLA
}

\author{
GUILHERME M. BURKHARDT* \\ MARCELO K. LENZI**
}

\begin{abstract}
O azeite de oliva possui alta qualidade nutricional devido aos benefícios para a saúde relacionados aos seus componentes, que atuam principalmente na prevenção de doenças coronarianas. Devido ao seu alto preço quando puro, o azeite é muitas vezes alvo de adulteração. Estes geralmente ocorrem adicionando óleos vegetais menos nobres como o soja. Este trabalho visa aplicar a análise de imagens para avaliação quantitativa da mistura de azeite de oliva e óleo de canola, combinando informações do espectro de absorbância UV-visível e de imagens digitais a partir de sua decomposição de cores, associando as características físicas destas misturas. Para fins de modelagem, foram utilizadas onze misturas de óleo de oliva e canola com fração mássica de azeite de oliva variando de 0 a $100 \%$ em intervalos de 10\%. Para fins de validação, uma amostra independente de $25 \%$ da fração mássica de azeite de oliva foi considerada. Para este estudo, foram desenvolvidos modelos lineares considerando todas as combinações possíveis entre as variáveis independentes: os componentes de cor $R$ (vermelho), G (verde), B (azul) e a absorbância nos comprimentos de onda selecionados $415 \mathrm{~nm}$, 440nm e $670 \mathrm{~nm}$. Considerando os resultados obtidos, observa-se que todos os modelos descrevem adequadamente os dados experimentais em função do ajuste do modelo e validação estatística. Concluiu-se que os modelos apresentados levaram a uma previsão muito próxima da amostra de teste em que o uso dos componentes $R, G$ e B da imagem digital associada à UV-Visible representam uma informação mais global sobre a amostra, o que resulta na melhor previsão da amostra de validação $\operatorname{como}(24,4 \pm 0,6) \%$.
\end{abstract}

\footnotetext{
*Mestre em Eng. Química, Universidade Federal do Paraná, Curitiba, PR (e-mail: guilherme.burkhardt@ hotmail.com).

**Doutor em Eng. Química, Universidade Federal do Paraná, Curitiba, PR (e-mail: lenzi@ufpr.br).
} 


\section{INTRODUÇÃO}

O azeite de oliva possui uma alta qualidade nutricional devido aos benefícios de saúde relacionados aos componentes deste óleo, os quais atuam principalmente na prevenção de doenças coronarianas. (FERRARA, LA; et al., 2000, COVAS, MI; et al, 2006). Os benefícios associados ao consumo do azeite de oliva estão diretamente relacionados à sua composição, que é rica em ácidos graxos monoinsaturados, como o ácido oleico (56 a 84\%), e baixo em saturados. Devido ao seu alto preço quando puro, o azeite frequentemente é alvo de adulterações. Estas usualmente ocorrem pela adição de óleos vegetais menos nobres, como o de soja. (FERNANDES et al., 2013, LICODIEDOFF et al., 2013)

Para detectar este tipo de fraude muitas técnicas analíticas têm sido empregadas e, apesar das vantagens de cada uma delas, todas necessitam de procedimentos de preparação de amostras, os quais exigem a manipulação dos produtos a serem analisados (GONILELLI, et al., 2013, FERNANDES, 2011).

Desde o advento dos computadores digitais na década de 1960, e seu enorme potencial para a realização de tarefas de análise de imagem começou a se tornar claras as tentativas e muitos foram os feitos para isso. Um sucesso inicial foi o notável reconhecimento óptico de caracteres, e seguindo os anos surgiu o reconhecimento da análise de impressões digitais, juntamente com um grande número de aplicações médicas.

Durante a última década, a vigilância tem sido objeto de muitos estudos e algoritmos bastante sofisticados foram desenvolvidos para o rastreamento de veículos e pessoas (rastreio por um lado, para controlar o tráfego e para garantir a segurança nas estradas, e por outro para a detecção ou mesmo antecipar crimes em tempo real) (DAVIS, 2009).

Até o recente advento dos métodos de análise instrumental, a grande maioria dos ensaios foram realizados com base nos ensaios colorimétricos à base de reagente, quer sob a forma de testes no local qualitativos ou quantitativos titulações. Enquanto os métodos instrumentais substituíram quase completamente titulações, testes no local mantém sua popularidade como métodos de rastreio, devido à sua facilidade de uso e baixo custo operacional, combinado com a capacidades de alto rendimento (BYRNE et al., 2000).

Recentemente, tem havido um aumento rápido na tecnologia da fotografia digital, tanto em termos de hardware e software. Os fortes indícios são de que este rápido desenvolvimento vai continuar nos próximos anos, impulsionado pelo baixo custo, aquisição de imagem de alto desempenho para produtos de consumo e da Web. A combinação da fotografia digital e ensaios colorimétricos potencialmente oferece uma rota para 0 alto rendimento qualitativo e medições analíticas quantitativas (BYRNE et al, 2000).

Vários trabalhos têm sido relatados na literatura a fim de explorar a utilização da cromatografia em fase gasosa, cromatografia líquida, ressonância magnética nuclear, infravermelho com transformada de Fourier, voltametria de onda quadrada, e calorimetria de varrimento diferencial, entre outros, para o controle da qualidade dos óleos vegetais. No entanto, a maior parte dos métodos são altamente sofisticados, caros e laboriosos.

Hoje em dia, a imagem digital é cada vez mais importante devido à sua capacidade para realizar a análise de baixo custo de maneira rápida e não-invasiva nos alimentos. Na verdade, uma grande variedade de câmeras digitais e equipamentos de digitalização tem contribuído para aumentar o número de artigos publicados explorando o uso de webcam, scanner, telefones celulares e câmera digital para monitorar a qualidade de várias amostras de alimentos. Além disso, outra característica positiva do uso de imagem digital para monitorar a qualidade dos alimentos é que ela substitui o sistema visual humano, muitas vezes empregado neste tipo de análises. Portanto, a utilização da imagem digital elimina o carácter subjetivo de análises, bem como a dependência do sistema visual humano, que é substancialmente influenciado por condições ambientais e sujeito a inconsistências (MILANEZ \& PONTES, 2013). 
Levando em consideração a necessidade de controle de qualidade para atender a legislação vigente na Resolução RDC $n^{\circ} 270$, de 22 de setembro de 2005 que determina a classificação dos óleos vegetais, utilizando métodos de baixo custo e rápida análise, minimizando os erros gerados por pessoas e suas impressões sobre os aspectos relacionados aos alimentos, em que alguns casos o olho humano não é tão sensível ao ponto de distinguir produtos in natura e processados/ adulterados, o presente trabalho visa utilizar a análise de imagem digital, para identificar características em misturas de azeite de oliva e óleo de canola.

\section{MATERIAIS E MÉTODOS}

\subsection{MATERIAL}

As matérias primas foram utilizadas em sua forma comercial, sem nenhum tratamento prévio, sendo: Azeite de Oliva Extra Virgem (acidez máxima $\leq 0,5 \%$, índice de peróxidos (mEq/ $\mathrm{Kg}$ ): $\leq 20.0$ ) da marca Gallo, produzido e Envasado por: Victor Guedes, Ind. Com. S.A. na cidade Abrantes, Pais Portugal, fabricado em 05/2014 sendo o número do lote 412950096 com a validade até 11/2015 e 100\% Óleo Refinado de Canola da marca Liza, produzido e Envasado por: Cargil Agricola S.A. cidade de Mairinque estado de São Paulo, Pais Brasil, fabricado em 09/05/2014 sendo o número do lote $05 \mathrm{C}$ com a validade até $09 / 05 / 2015$. Todas as amostras foram provenientes de um único lote e num curto intervalo de tempo.

\subsection{PREPARAÇÃO DAS AMOSTRAS}

Inicialmente, prepararam-se as amostras para análise em cubetas de poliestireno de face ótica de $1 \mathrm{~cm}$, sempre se baseando em sua fração mássica, onde as matérias primas, azeite de oliva e canola, foram misturadas com uma variação de $0 \%$ a $100 \%$ em massa de azeite de oliva em oleo de canola respectivamente com intervalo de $10 \%$, sendo então, onze amostras de concentração mássica diferentes. Desta forma, a amostra 1 possui apenas azeite de oliva, a amostra 2 possui $90 \%$ em massa de azeite de oliva e 10\% em massa de óleo de canola, a amostra 3 possui $80 \%$ em massa de azeite de oliva e $20 \%$ em massa de óleo de canola e assim sucessivamente, até a amostra 10 que possui $10 \%$ em massa de azeite de oliva e $90 \%$ em massa de óleo de canola e a amostra 11 que possui apenas óleo de canola.

Além destas onze amostras, mais uma foi preparada contendo uma concentração conhecida de $25 \%$ em massa de azeite de oliva e $75 \%$ em massa de óleo de canola, a qual será utilizada para validação do sistema, totalizando assim doze amostras ao final do estudo.

\subsection{AQUISIÇÃO DE IMAGENS}

Após a realização das amostras foi feita a aquisição das imagens, com uma máquina fotográfica digital Marca Sony®, modelo DSC-H100 fabricado no Polo Industrial de Manaus, Amazonas, Brasil, Número de série 8111984, com uma resolução de 5 megapixels, zoom de 1,4x, ISO 80 e abertura 4:3 em modo luz ambiente. Para tentar minimizar o fator iluminação sobre as amostras, utilizou-se uma câmara de coloração interna branca, onde a fonte de iluminação foi constante durante a tomada da fotografia.

Para a análise de imagem dos valores de cores no sistema RGB para todas as amostras em questão, foi desenvolvido um algoritmo no programador Delphi 6, facilitando a aquisição dos dados, ressaltando que a imagem não sofreu nenhum pós-processamento.

\subsection{ESPECTOMETRIA E MODELAGEM}

Posteriormente, foi obtido um espectro de varredura (190nm a 900nm) de todas as doze amostras, utilizando: Equipamento Espectrômetro UV - Visível, Marca Cary modelo 100 Scan em 
uma faixa de varredura de 310 a 900nm, num intervalo de amostragem de $1 \mathrm{~nm}$ do Departamento de Química da Universidade Federal do Paraná.

Foram selecionadas para a análise dos resultados as absorbâncias de três comprimentos de onda referentes aos picos do espectro das amostras, os quais apresentaram maior desvio padrão entre si. parâmetros).

Os dados foram tratados estatisticamente pelo Software Statistica (estimação de

\section{RESULTADOS E DISCUSSÃO}

\subsection{DECOMPOSIÇÃO E APRESENTAÇÃO DOS DADOS EM RGB}

A obtenção das componentes R, G, B foram feitas através da decomposição da cor de uma área de referência da imagem de cada uma das cubetas, $A$ Tabela 1 apresenta a estatística descritiva básica das componentes R, G, B e os valores das absorbâncias nos comprimentos de onda de dois picos (415 e $670 \mathrm{~nm}$ ) e um vale e $440 \mathrm{~nm}$.

\section{TABELA 1 ESTATÍSTICA DOS VALORES DAS COMPONENTES R,G,B E OS VALORES DAS ABSORBÂNCIAS PARA CADA UMA DAS AMOSTRAS.}

\begin{tabular}{cccccccccc}
\hline $\begin{array}{l}\text { \% Oliva na } \\
\text { amostra }\end{array}$ & $\begin{array}{c}\mathrm{R} \\
\text { médio }\end{array}$ & $\begin{array}{c}\mathrm{R} \\
\text { desvio }\end{array}$ & $\begin{array}{c}\mathrm{G} \\
\text { médio }\end{array}$ & $\begin{array}{c}\mathrm{G} \\
\text { desvio }\end{array}$ & $\begin{array}{c}\mathrm{B} \\
\text { médio }\end{array}$ & $\begin{array}{c}\mathrm{B} \\
\text { desvio }\end{array}$ & $\begin{array}{c}\mathrm{ABS} \\
415 \mathrm{~nm}\end{array}$ & $\begin{array}{c}\mathrm{ABS} \\
440 \mathrm{~nm}\end{array}$ & $\begin{array}{c}\mathrm{ABS} \\
670 \mathrm{~nm}\end{array}$ \\
100 & 114 & 2 & 121 & 2 & 5 & 1 & 3,377 & 2,518 & 1,118 \\
90 & 117 & 2 & 124 & 2 & 5 & 2 & 3,037 & 2,178 & 0,923 \\
80 & 120 & 2 & 128 & 3 & 11 & 4 & 2,761 & 1,952 & 0,800 \\
70 & 122 & 3 & 130 & 3 & 15 & 5 & 2,478 & 1,740 & 0,718 \\
60 & 123 & 4 & 132 & 4 & 16 & 7 & 2,162 & 1,505 & 0,618 \\
50 & 123 & 4 & 133 & 4 & 24 & 9 & 1,859 & 1,289 & 0,515 \\
40 & 128 & 2 & 139 & 2 & 45 & 4 & 1,519 & 1,046 & 0,417 \\
30 & 132 & 2 & 144 & 3 & 73 & 5 & 1,247 & 0,858 & 0,340 \\
20 & 134 & 2 & 147 & 2 & 102 & 3 & 0,917 & 0,629 & 0,230 \\
10 & 132 & 3 & 143 & 2 & 127 & 4 & 0,695 & 0,503 & 0,217 \\
0 & 131 & 3 & 144 & 3 & 167 & 3 & 0,269 & 0,170 & 0,034 \\
\hline
\end{tabular}

Na Figura 1 ilustra o comportamento desta variável $R$ em função do aumento da concentração de azeite de oliva na mistura.

Os resultados referentes à cor $G$ podem ser vistos na Figura 2. Em relação ao componente $B$, os resultados estão expressos graficamente na Figura 3. Ao serem considerados os comportamentos em função da concentração do azeite de oliva na mistura, observa-se que o componente $R$ e $o$ componente $G$ possuem um maior grau de linearidade ao longo de sua faixa de variação, quando comparado ao componente B. 


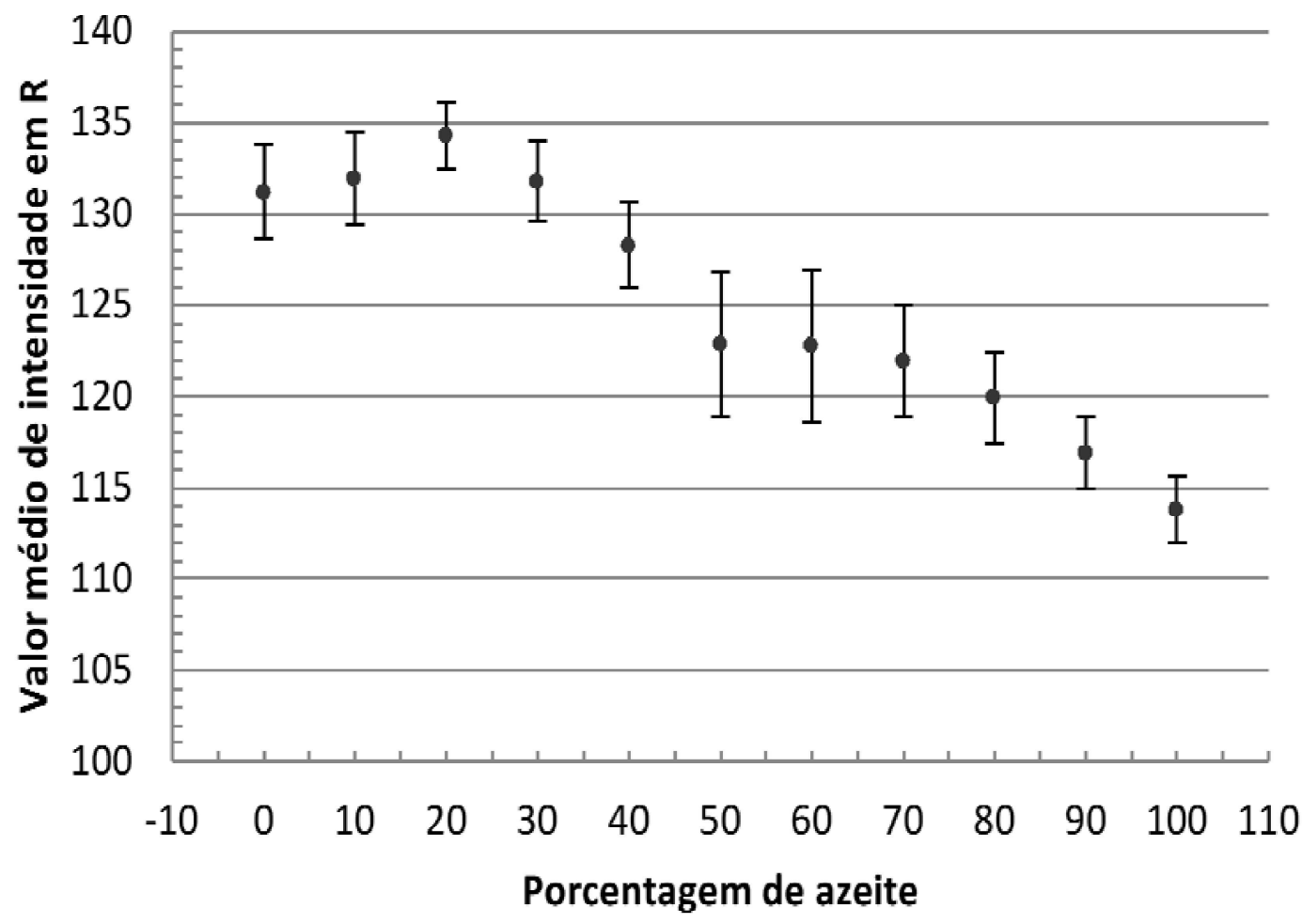

FIGURA 1 COMPORTAMENTO DOS VALORES DECOMPOSTOS EM R (VERMELHO) DA IMAGEM DAS AMOSTRAS PARA CADA UMA DAS CONCENTRAÇÕES MÁSSICAS.

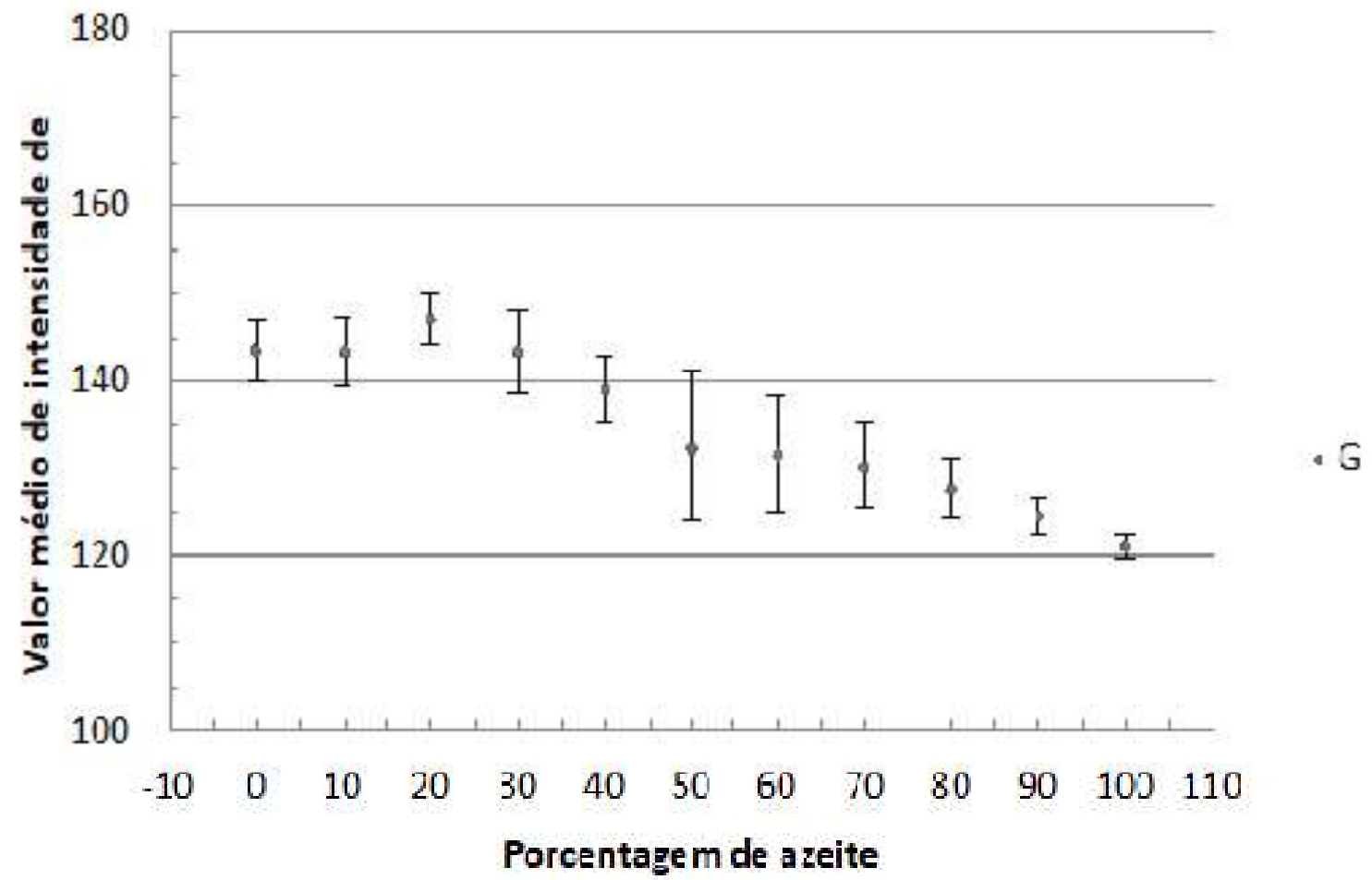

FIGURA 2 COMPORTAMENTO DOS VALORES DECOMPOSTOS EM G (VERDE) DA IMAGEM DAS AMOSTRAS PARA CADA UMA DAS CONCENTRAÇÕES MÁSSICAS. 


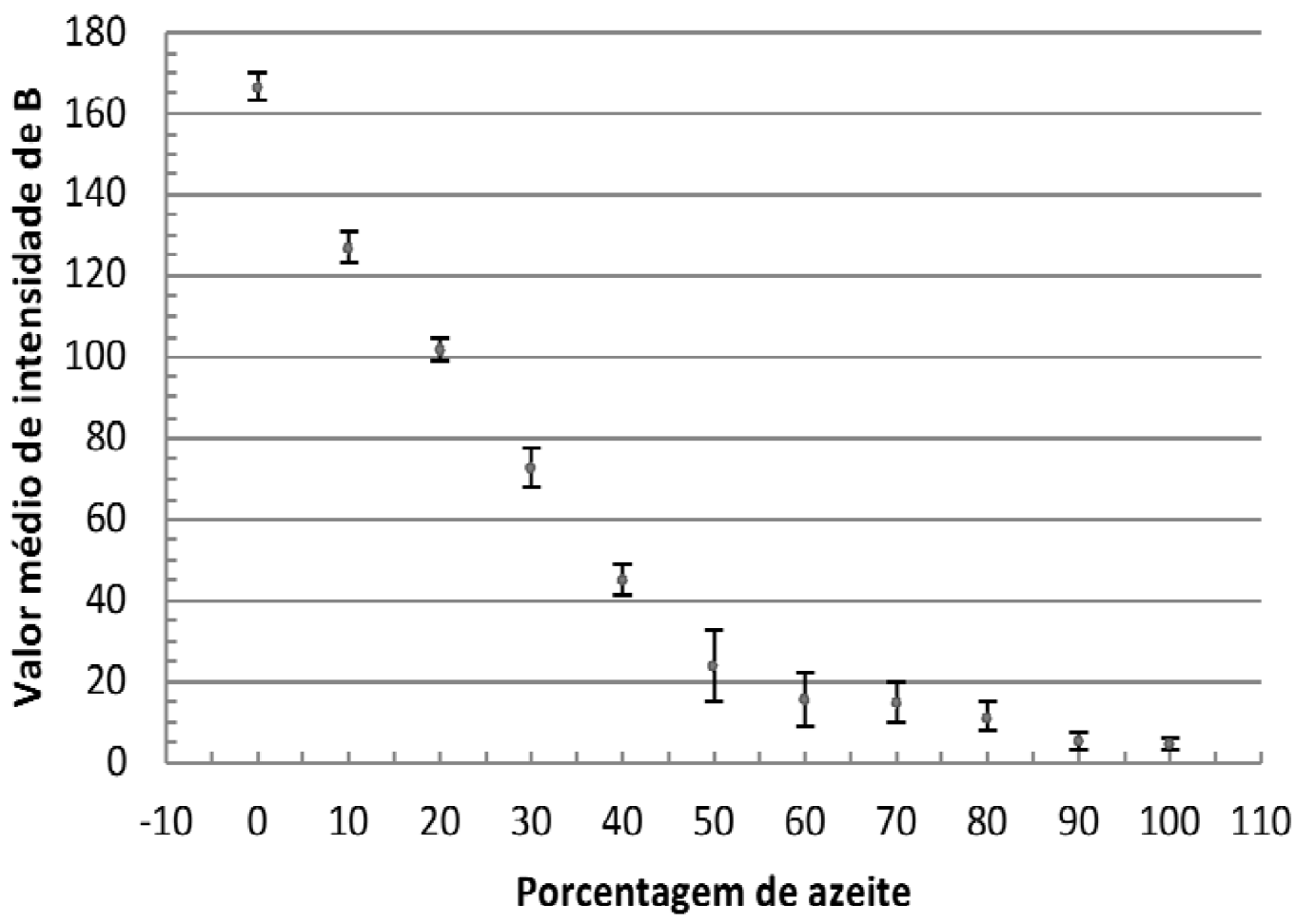

FIGURA 3 COMPORTAMENTO DOS VALORES DECOMPOSTOS EM B (AZUL) DA IMAGEM DAS AMOSTRAS PARA CADA UMA DAS CONCENTRAÇÕES MÁSSICAS.

As amostras das misturas foram analisadas em um espectrofotometro de UV-VIS e apresentadas na Tabela 1 para complementar a caracterização e posteriormente a modelagem preditiva das misturas entre azeite de oliva e canola. A Figura 4 apresenta os espectros brutos para todas as composições. Para fins de modelagem, foram selecionados dois picos e um vale em comprimentos de onda cuja absorbância apresentasse maior variância (amplitude), indicando uma melhor diferenciação e evitando sobreposição de informações o mesmo que fora observado no trabalho proposto por Fernandes (2011).

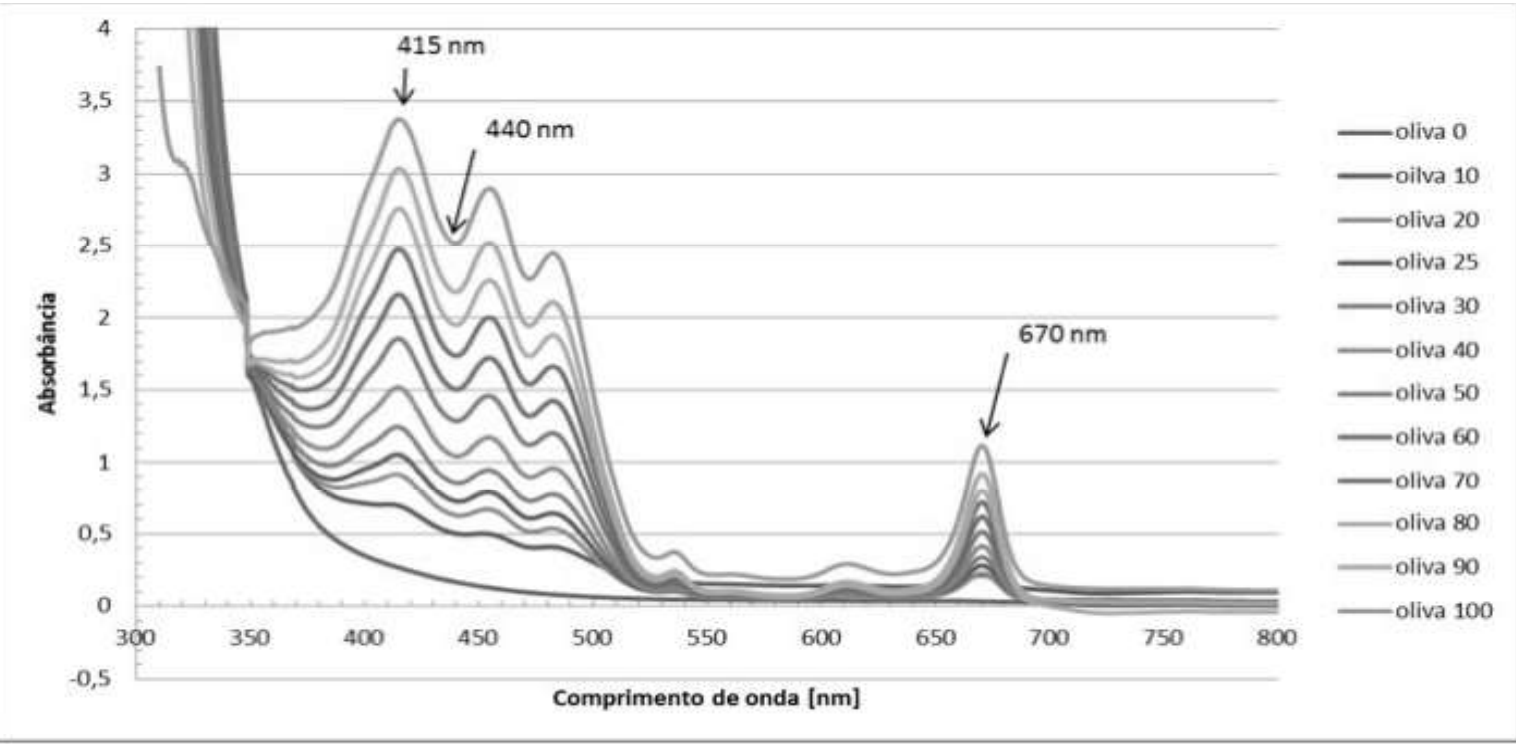

FIGURA 4 GRÁFICO DAS AMOSTRAS APRESENTADAS ANALISADAS EM UM ESPECTROFOTÔMETRO DE UV-VIS DE VARREDURA. 
Os estudos de modelagem tiveram por objetivo a determinação de equações capazes de descrever o percentual de azeite de oliva nas misturas a partir das componentes $R, G, B$ e as absorbâncias nos comprimentos de onda $415 \mathrm{~nm}, 440 \mathrm{~nm}$ e $670 \mathrm{~nm}$. Desta forma, torna-se importante avaliar a correlação entre as variáveis. A Figura 5 apresenta a matriz de gráficos de correlação, ao passo que a Tabela 2 apresenta os valores dos coeficientes de correlação entre as variáveis.

\section{TABELA 2 APRESENTAÇÃO DOS VALORES DOS COEFICIENTES DE CORRELAÇÃO ENTRE AS VARIÁVEIS.}

\begin{tabular}{cccccccc}
\hline & Massa & ABS 145 & ABS 440 & ABS 670 & $R$ & $G$ & $B$ \\
Massa & 1,00 & 1,00 & 1,00 & 0,99 & $-0,95$ & $-0,96$ & $-0,92$ \\
ABS 415 & 1,00 & 1,00 & 1,00 & 0,99 & $-0,95$ & $-0,96$ & $-0,93$ \\
ABS 440 & 1,00 & 1,00 & 1,00 & 1,00 & $-0,95$ & $-0,96$ & $-0,91$ \\
ABS 670 & 0,99 & 0,99 & 1,00 & 1,00 & $-0,95$ & $-0,96$ & $-0,89$ \\
R & $-0,95$ & $-0,95$ & $-0,95$ & $-0,95$ & 1,00 & 1,00 & 0,83 \\
G & $-0,96$ & $-0,96$ & $-0,96$ & $-0,96$ & 1,00 & 1,00 & 0,83 \\
B & $-0,92$ & $-0,93$ & $-0,91$ & $-0,89$ & 0,83 & 0,85 & 1,00 \\
\hline
\end{tabular}

Analisando os resultados, observa-se na Tabela 2 que a variável dependente, percentual mássico de azeite de oliva, apresenta forte correlação com as absorbâncias e os componentes da análise de imagem. Desta forma, modelos lineares entre estas variáveis devem ser capazes de fazer a predição adequada da composição da mistura observado visualmente na Figura 5.

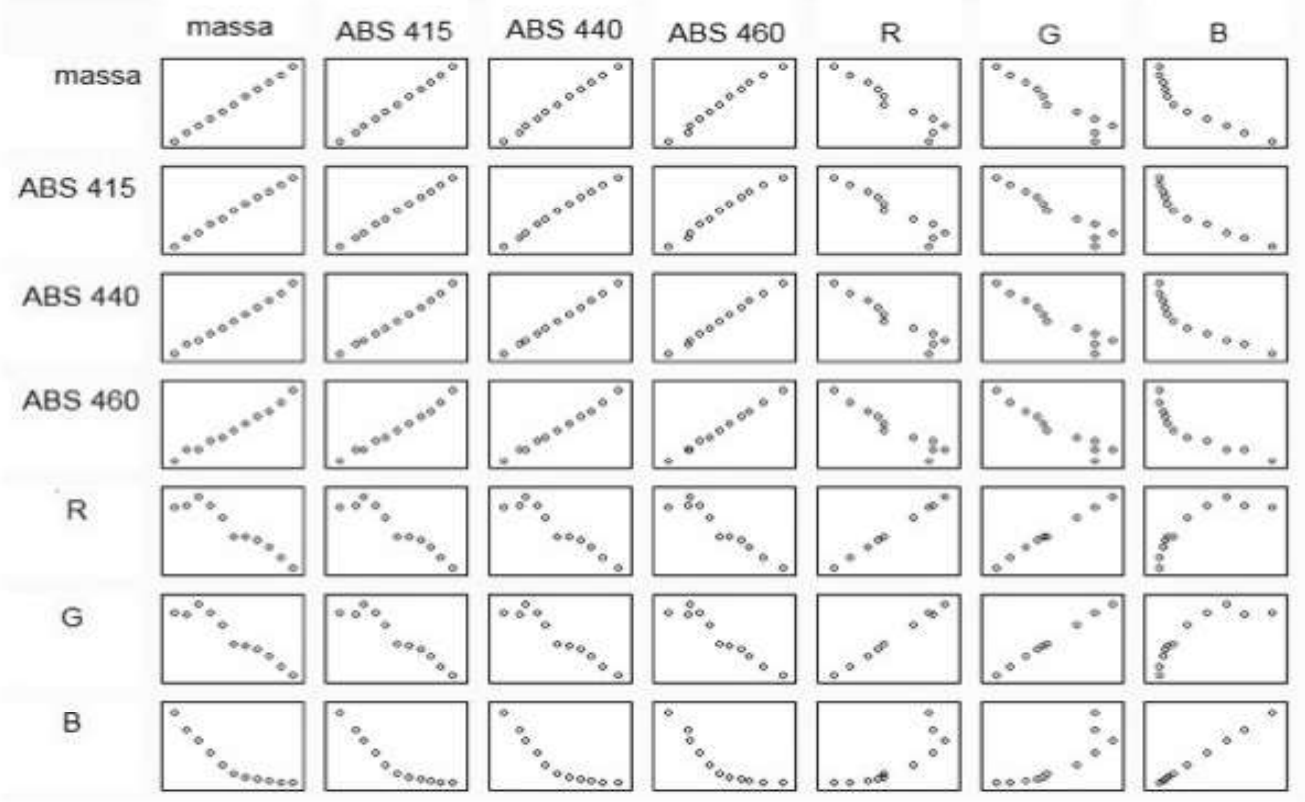

FIGURA 5 MATRIZ DE CORRELAÇÕES.

Os modelos matemáticos lineares foram desenvolvidos considerando todas as possíveis combinações entre as variáveis independentes, sendo os três componentes de cor R, G, B e as absorbâncias nos comprimentos de onda selecionados $415 \mathrm{~nm}$, 440nm e $670 \mathrm{~nm}$. A função objetivo para estimação de parâmetros foi a de mínimos quadrados, dada pela equação 07.

$$
F_{0}={ }^{N E} \sum_{(i=1)}\left(\text { massa }_{i}^{\text {EXP-massa }}{ }_{-i}^{\text {MOD }}\right)^{2}
$$

Onde Fo é a função objetivo baseada nos mínimos quadrados, NE é o número de experimento, massa ${ }^{\mathrm{EXP}}$ é o valor da concentração mássica teórica e massa ${ }^{\mathrm{MOD}}$ é o valor da concentração mássica modelado. 
A estimação de parâmetros foi feita no software STATISTICA, usando o pacote de estimação não linear. Em todas as simulações foi utilizado o método de Levenberg-Marquadt (LEVENBERG, 1944) e (MARQUARDT, 1963) sendo adotado um único critério de convergência dado por $10^{-6}$ e a estimativa inicial de todos os parâmetros foi $10^{-1}$.

Para análise, os modelos foram classificados conforme a quantidade de parâmetros, além de terem sido simuladas todas as combinações possíveis.

Sendo que as classes são utilizadas para diferenciar o conjunto de possíveis combinações, facilitando a diferenciação e a interpretação dos dados. Por exemplo, para modelos com dois parâmetros é possível obter as seguintes classes, resultando em 21 modelos:

Classe 1: massa $=a_{0}+a_{1} \cdot A B S_{p}$

(Total de 3 modelos)

Classe 2: massa $=a_{0} \cdot A B S_{p}+a_{1} \cdot A B S v$ sendo $p \neq v$

(Total de 3 modelos)

Classe 3: massa $=a_{0} \cdot A_{B S}+a_{1} \cdot C_{m}$

(Total de 9 modelos)

Classe 4: $m a s s a=a_{0} \cdot C_{m}+a_{1} \cdot C O R_{n}$ sendo $m \neq n$

(Total de 3 modelos)

Classe 5: massa $=a_{0}+a_{1} \cdot C_{0 R}$

(Total de 3 modelos)

Onde: $p, v$ : comprimento de onda $=415 \mathrm{~nm}, 440 \mathrm{~nm}$ e $670 \mathrm{~nm} \mathrm{~m}$, n: as cores R (Red); $\mathrm{G}($ Green); B(Blue)

Para modelos com três parâmetros, obtém-se 35 modelos divididos em:

Classe 1: massa $=a_{0}+a_{1} \cdot \operatorname{COR}_{m}+a_{2} \operatorname{COR}_{n}$

(Total de 3 modelos)

Classe 2: massa $=a_{0}+a_{1} \cdot C_{m}+a_{2} \cdot A \cdot S_{p}$

(Total de 9 modelos)

Classe 3: massa $=a_{0}+a_{1} \cdot A B S_{p}+a_{2} \cdot A B S_{q}$

(Total de 3 modelos)

Classe 4: massa $=a_{0} \cdot \operatorname{COR}_{\mathrm{m}}+\mathrm{a}_{1} \cdot \mathrm{COR}_{\mathrm{n}}+\mathrm{a}_{2} \cdot \mathrm{COR}_{\mathrm{s}}$

(Total de 1 modelos)

Classe 5: massa $=a_{0} \cdot \operatorname{COR}_{\mathrm{m}}+\mathrm{a}_{1} \cdot \mathrm{COR}_{\mathrm{n}}+\mathrm{a}_{2} \cdot \mathrm{ABS}_{\mathrm{p}}$

(Total de 9 modelos)

Classe 6: massa $=a_{0} \cdot \operatorname{COR}_{m}+a_{1} \cdot A B S_{p}+a_{2} \cdot A B S_{q}$

(Total de 9 modelos)

Classe 7: massa $=a_{0} \cdot A B S_{p}+a_{1} \cdot A B S_{q}+a_{2} \cdot A B S_{0}$

(Total de 1 modelos)

Onde: "p" e "v" são os comprimentos de onda (415nm, 440nm e 670nm); "m", "n", "s" são as cores da imagem conforme o sistema RGB, R (Red); G(Green); B(Blue)

TABELA 3 LISTA DOS MELHORES MODELOS COMPOSTOS POR DOIS PARÂMETROS.

Classe 1 Modelo 1

Classe 3 Modelo 1

massa $=a_{0}+a_{1} \cdot A B S 415$

massa $=a_{0} \cdot A B S 15+a_{1} \cdot R$

Classe 3 Modelo 2

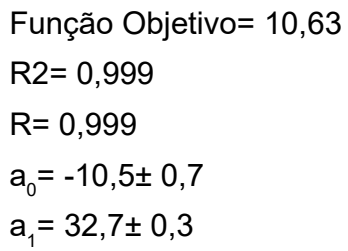

Covariância entre $a_{0}$ e $a_{1}=-0,21$

Função Objetivo $=10,24$

$\mathrm{R} 2=0,999$

$\mathrm{R}=0,9995$

$a_{0}=32,3 \pm 0,3$

$a_{1}=-0,1 \pm 0,0$

Covariância entre $a_{0}$ e $a_{1}=-0,077$

Função Objetivo $=10,55$

$\mathrm{R} 2=0,999$

$\mathrm{R}=0,9995$

$a_{0}=32,2 \pm 0,3$

$a_{1}=-0,1 \pm 0,0$

Covariância entre $a_{0}$ e $a_{1}=-0,001$ 


\begin{tabular}{|c|c|}
\hline Classe 3 Modelo 3 & \\
\hline \multirow{9}{*}{ massa $=a_{0}+a_{1} \cdot A B S 440+a_{2} \cdot A B S 670$} & Função Objetivo= 5,593 \\
\hline & $\mathrm{R} 2=0,9995$ \\
\hline & $R=0,9997$ \\
\hline & $a_{0}=-12,1 \pm 0,7$ \\
\hline & $a_{1}=85,7 \pm 5,8$ \\
\hline & $a_{2}=-92,8 \pm 12,9$ \\
\hline & Covariância entre $a_{0}$ e $a_{1}=-3,291$ \\
\hline & Covariância entre $a_{0}$ e $a_{2}=7,033$ \\
\hline & Covariância entre $a_{1}$ e $a_{2}=-74,225$ \\
\hline \multicolumn{2}{|l|}{ Classe 6 Modelo 4} \\
\hline \multirow{9}{*}{ massa $=a_{0} \cdot R+a_{1} \cdot A B S 670+a_{2} \cdot A B S 440$} & Função Objetivo= 5,3744 \\
\hline & $R 2=0,9995$ \\
\hline & $R=0,9997$ \\
\hline & $a_{0}=-0,1 \pm 0,0$ \\
\hline & $a_{1}=-93,7 \pm 12,7$ \\
\hline & $a_{2}=85,3 \pm 5,6$ \\
\hline & Covariância entre $a_{0}$ e $a_{1}=0,050$ \\
\hline & Covariância entre $a_{0}$ e $a_{2}=-0,023$ \\
\hline & Covariância entre $a_{1}$ e $a_{2}=-71,281$ \\
\hline \multicolumn{2}{|l|}{ Classe 6 Modelo 5} \\
\hline \multirow{9}{*}{ massa $=a_{0} \cdot G+a_{1} \cdot A B S 670+a_{2} \cdot A B S 440$} & Função Objetivo= 5,539 \\
\hline & $R 2=0,9995$ \\
\hline & $R=0,9997$ \\
\hline & $a_{0}=-0,1 \pm 0,0$ \\
\hline & $a_{1}=165,9 \pm 12,9$ \\
\hline & $a_{2}=32,4 \pm 5,7$ \\
\hline & Covariância entre $a_{0}$ e $a_{1}=0,047$ \\
\hline & Covariância entre $a_{0}$ e $a_{2}=-0,022$ \\
\hline & Covariância entre $a_{1}$ e $a_{2}=-73,224$ \\
\hline
\end{tabular}

A Tabela 3 apresenta as três melhores simulações referentes a modelos de dois parâmetros. Na Tabela 4 é apresenta as três melhores simulações referentes aos modelos de três parâmetros, as simulações foram dispostas por classe e modelos com a apresentação da análise. São apresentados também os valores estimados para os parâmetros, bem como a covariância paramétrica.

A Tabela 4 apresenta os resultados referentes aos melhores modelos com três parâmetros. Em função do maior número de parâmetros, espera-se um menor valor da função objetivo para estes modelos, destaca-se que modelos envolvendo os componentes da análise de imagem $R, G$ e $B$ levam à parâmetros com menor desvio padrão percentual.

Observa-se que o uso dos componentes da análise de imagem acoplada à absorbância é mais atrativo do que apenas o uso de valores de absorbância para a predição da composição das misturas.

\subsection{APRESENTAÇÃO DOS RESULTADOS DAS PREDIÇÕES}

A seguir os gráficos relacionam os valores observado vs predito e de normalidade dos melhores modelos obtidos. 


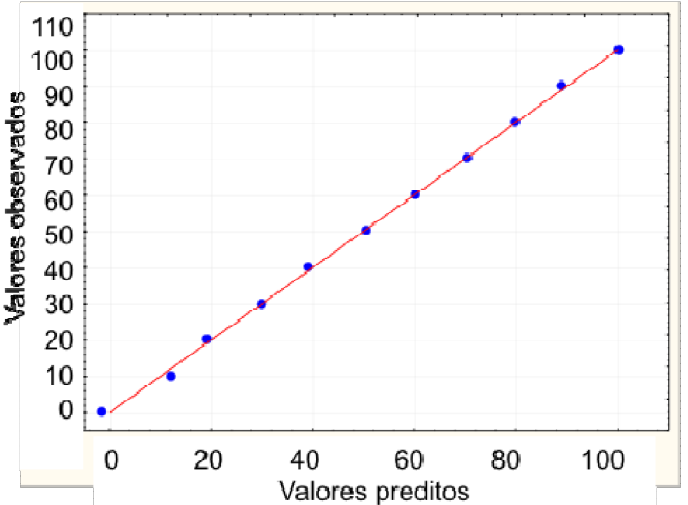

(a)

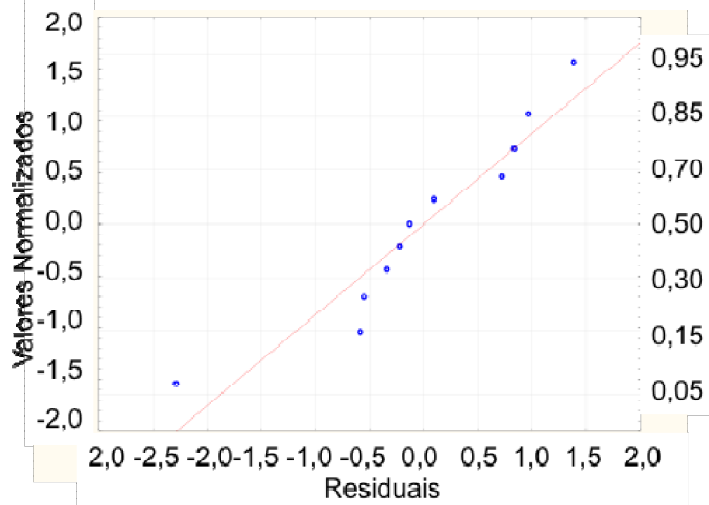

(b)

FIGURA 6 O GRÁFICO (A) RELACIONA OS VALORES OBSERVADOS VERSOS OS PREDITOS PELO MODELO CLASSE3 MODELO 1 COM DOIS PARÂMETROS E O GRÁFICO (B) A DISTRIBUIÇÃO NORMAL DOS RESÍDUOS.

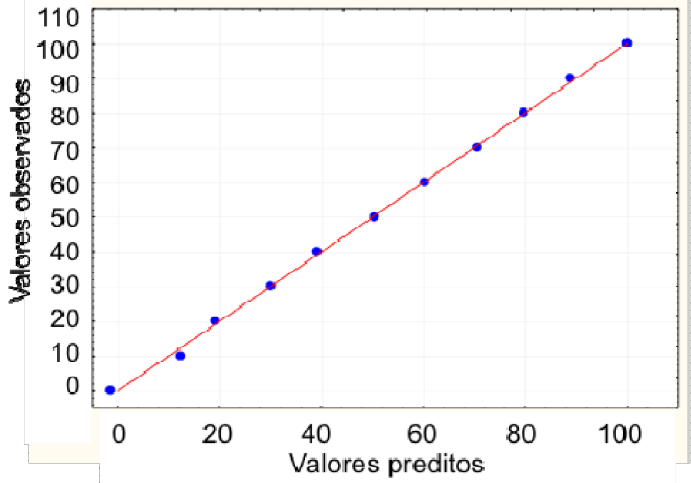

(a)

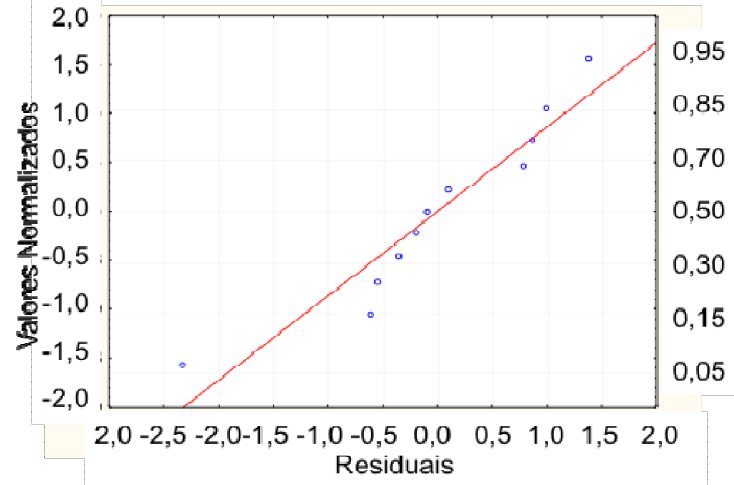

(b)

FIGURA 7 O GRÁFICO (A) RELACIONA OS VALORES OBSERVADOS VERSOS OS PREDITOS PELO MODELO CLASSE3 MODELO 2 COM DOIS PARÂMETROS E O GRÁFICO (B) A DISTRIBUIÇÃO NORMAL DOS RESÍDUOS.

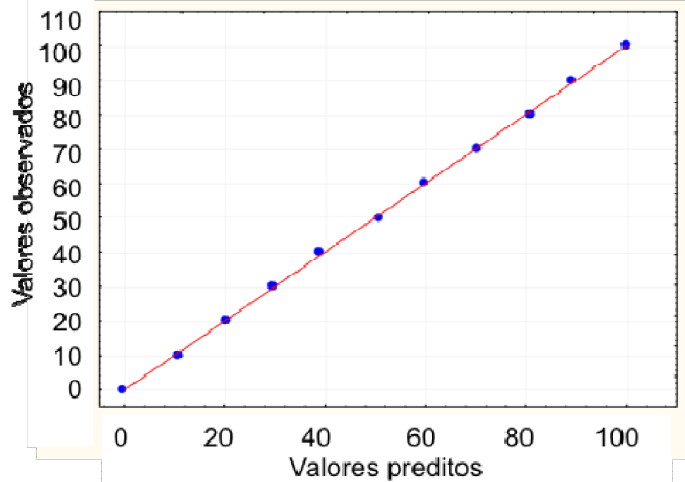

(a)

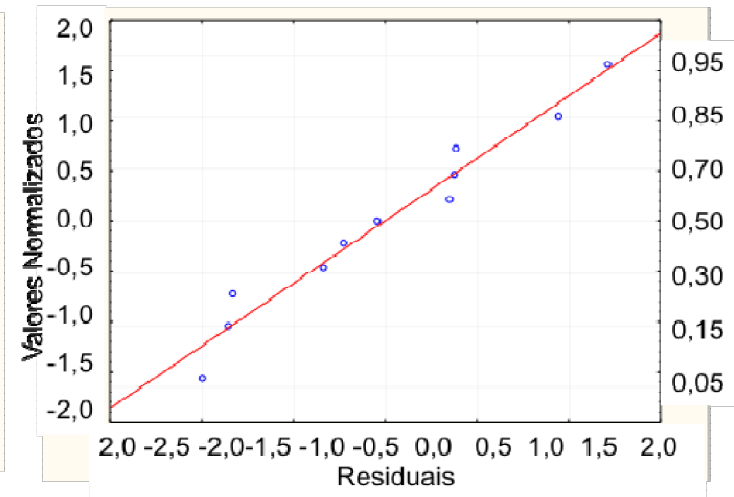

(b)

FIGURA 8 O GRÁFICO (A) RELACIONA OS VALORES OBSERVADOS VERSOS OS PREDITOS PELO MODELO CLASSE6 MODELO 4 COM TRÊS PARÂMETROS E O GRÁFICO (B) A DISTRIBUIÇÃO NORMAL DOS RESÍDUOS. 
Após a análise dos gráficos observa-se que todos os modelos apresentam boa representatividade para os dados experimentais tendo em vista que os valores preditos são muito próximos dos experimentais como podem ser vistos nos gráficos (a) uma vez que os pontos situamse muito próximos da reta de inclinação $45^{\circ}$. Além disso, observa-se que os resíduos tendem a seguir uma distribuição normal como pedem ser observados no gráficos (b), isso é importante pois esta "normalidade" dos resíduos caracteriza a ausência de erros sistemáticos, assim eventuais diferenças entre dados experimentais e predições do modelo surgem em função de possíveis erros aleatórios inerentes a medição experimental.

\subsection{VALIDAÇÃO DOS MODELOS}

Com intuito de validação da técnica, foi feita uma mistura teste com composição $25 \%$ em massa de azeite de oliva em óleo de canola a qual também foi fotografada e analisada com o software para decomposição de cor nos componentes $\mathrm{R}, \mathrm{G}$ e B, bem como foi realizada uma análise espectrofotométrica de UV-VIS. Os dados referentes à análise estatística da imagem são apresentados na Tabela 5. O desvio-padrão da predição do modelo foi calculado com base em ISFER et al. (2010).

\section{TABELA 5 DADOS PARA VALIDAÇÃO DO MODELO (25\% DE MASSA DE AZEITE DE OLIVA)}

\begin{tabular}{cccccccccc}
\hline $\begin{array}{c}\text { \%Oliva } \\
\text { na }\end{array}$ & $\begin{array}{c}\mathrm{R} \\
\text { medio }\end{array}$ & $\begin{array}{c}\mathrm{R} \\
\text { desvio }\end{array}$ & $\begin{array}{c}\mathrm{G} \\
\text { medio }\end{array}$ & $\begin{array}{c}\mathrm{G} \\
\text { desvio }\end{array}$ & $\begin{array}{c}\mathrm{B} \\
\text { medio }\end{array}$ & $\begin{array}{c}\mathrm{B} \\
\text { desvio }\end{array}$ & $\begin{array}{c}\text { ABS } \\
415\end{array}$ & $\begin{array}{c}\text { ABS } \\
440\end{array}$ & $\begin{array}{c}\text { ABS } \\
670\end{array}$ \\
25 & 125 & 2 & 138 & 3 & 79 & 4 & 1,055 & 0,727 & 0,283 \\
\hline
\end{tabular}

As predições e os respectivos desvios padrão são apresentados na Tabela 6 para os modelos de dois parâmetros e três parâmetros. Ressalta-se que os valores das variáveis independentes ( $R$, G, B, ABS415, ABS440, ABS670) foram considerados com desvio padrão desprezíveis.

TABELA 6 VALORES DAS PREDIÇÕES EM \% E DOS RESPECTIVOS DESVIOS PADRÕES NA FORMA DE DUAS VEZES O INTERVALO DE CONFIANÇA $S_{\text {MASSA }}$ PARA: DOIS PARÂMETROS (2PAR), TRÊS PARÂMETROS (3PAR), CLASSE 1 (CLA1), CLASSE 3 (CLA3), CLASSE 6 (CLA6), MODELOS 1 (MOD1), MODELOS 2 (MOD2), MODELOS 4 (MOD4), MODELOS 5 (MOD5).

\begin{tabular}{ccc}
\hline Modelos & Valor & erro \\
2 PARCLA1MOD1 & 24,0 & 0,8 \\
2 PARCLA3MOD1 & 24,4 & 0,8 \\
2 PARCLA3MOD2 & 24,3 & 0,8 \\
3 PARCLA3MOD3 & 23,9 & 0,7 \\
3 PARCLA6MOD4 & 24,4 & 0,6 \\
3 PARCLA6MOD5 & 24,2 & 0,6 \\
\hline
\end{tabular}

A Figura 12 apresenta a comparação das predições do modelo para amostra de $25 \%$. Observa-se que os modelos, Classe 3 - Modelo 1 e 2 de 2 parâmetros e os modelos Classe 3 Modelo 3 e Classe 6 Modelo 4 de 3 parâmetros contêm o valor teórico da predição em seu intervalo considerando duas vezes o desvio padrão. Podemos dizer que os modelos Classe 3 Modelo 1 - 2 parâmetro e Classe 6 Modelo 4 de -3 parâmetros apresentam melhores resultados pois, se aproximam mais do valor de 25. Logo o modelo Classe 6 Modelo 4 de 3 parâmetro apresenta menor valor da função objetivo, o que caracteriza predições com maior verossimilhança. 


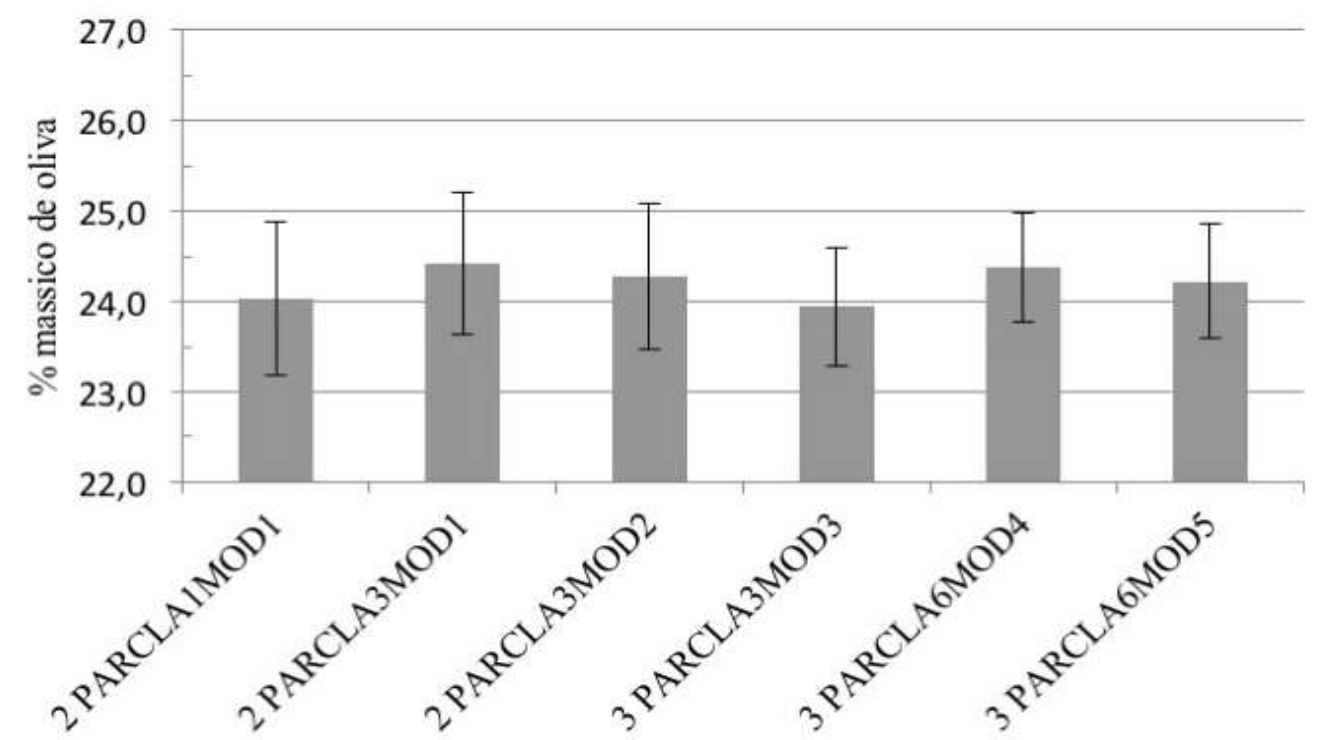

\section{FIGURA 9 COMPARAÇÃO DAS PREDIÇÕES DOS MODELOS PARA A AMOSTRA TESTE DE 25\% DE AZEITE EM SUA COMPOSIÇÃO MÁSSICA.}

Observa-se com base nos resultados obtidos que o uso da análise das absorbâncias associada a imagem digital permitiu uma melhor caracterização da composição de misturas de azeite de oliva e óleo de canola, quando comparado apenas ao uso de medições de absorbância em análises espectrofotométricas de UV-VIS. Tal fato pode ser comprovado pela comparação das predições dos modelos com o valor referência de $25 \%$ as quais se aproximam muito ao valor predito.

\section{CONCLUSÃO}

O objetivo principal foi estudar a aplicação da análise de imagem para avaliação quantitativa da mistura de óleo de oliva e canola. Desenvolvendo modelos matemáticos lineares, fazendo uso de informações da combinação de dados, o espectro de absorbância na região do UV - visível e das imagens digitais relativos à decomposição das cores. Durante o estudo inicial para a determinação dos modelos com significado físico, observou-se que 3 modelos de dois e três parâmetros foram satisfatórios para o estudo proposto num universo de 56 modelos.

Escolhidos os modelos foram geradas simulações dos dados preditivos para uma amostra teste de $25 \%$ em massa de azeite de oliva em óleo de canola. A partir de uma visão geral do comportamento dos resultados para os modelos propostos, concluiu-se que os modelos gerados escolhidos são bons para predizer o valor da amostra teste, cujo o melhor valor foi de $(24,4 \pm 0,6) \%$ para modelo de Classe 6 Modelo 4 de três parâmetros, tal modelo também apresentou menor valor da função objetivo, bem como menores valores percentuais de desvio padrão paramétrico e também baixa covariância paramétrica. Logo o modelo da Classe 1 Modelo 1 de dois parâmetros, que considera apenas absorbância, apresenta um valor para função objetivo pequeno, no entanto, a variância e covariância paramétrica são consideráveis. Desta forma, observa-se que modelos mistos levaram à melhor predição, provavelmente pelo fato dos componentes $R, G$ e $B$ representarem uma informação mais globalizada sobre a amostra, diferentemente das absorbâncias que são específicas para cada comprimento de onda.

A partir deste estudo, abriu-se uma gama de possibilidades que poderão ser investigadas, tal como a construção de outros modelos para outras misturas, que visa utilizar a imagem digital para simular com maior realismo a quantificação de misturas de óleo vegetais e até estender para óleos combustíveis. Outra proposta seria o estabelecimento de uma metodologia utilizando imagens digitais, UV-Visível e Cromatografia Gasosa. 


\section{ABSTRACT}

\section{DIGITAL IMAGE USE FOR MEASUREMENT OF MIX OLIVE OIL AND OIL CANOLA}

Olive oil has a high nutritional quality due to the health benefits related to the components of this oil, which act mainly in the prevention of coronary heart disease. The benefits associated with olive oil consumption are directly related to its composition. Due to its high price when pure, olive oil is often tamper target. These usually occur by adding less noble vegetable oils such as soybean. This work aims at applying image analysis for quantitative assessment of olive and canola oil blend, making use of the data combining information the absorbance spectrum in the UV - visible and digital images on the decomposition of colors, associating the physical characteristics of these mixtures (olive oil/ canola oil). For modeling purposes, eleven mixtures of olive and canola oil with mass fraction of olive oil ranging from $0-100 \%$ by $10 \%$ were used. For validation purposes, a sample of $25 \%$ mass fraction of olive oil was considered. For this study, linear models were developed considering all possible combinations among the particular independent variables: the color components $\mathrm{R}$ (red), $\mathrm{G}$ (green), $B$ (blue) and the absorbance at selected wavelengths $415 \mathrm{~nm}, 440 \mathrm{~nm}$ and $670 \mathrm{~nm}$. Considering the obtained results, it is observed that all models adequately describe the experimental data due to the model fit and statistical validation. It was concluded that the presented models have led to very close prediction of the test sample in which the use of the components $R, G$ and $B$ digital image associated with the UV-Visible represent a more global information about the sample, which results in the best prediction was $(24.4 \pm 0.6) \%$.

KEYWORDS: IMAGE ANALYSIS, OLIVE OIL, CANOLA, MIXTURES AND SPECTROSCOPY.

\section{REFERÊNCIAS}

1 ALBUQUERQUE, M.P.; ALBUQUERQUE, M.P. Processamento de imagens: Métodos e Análises. Centro Brasileiro de Pesquisas Físicas -CBPF/MCT-RJ, 2002.

2 LICODIEDOFF, S; RIBANI, R.H.; CAMLOFSKI, A.M.D.; LENZI, M.K. Use of Image analysis for monitoring the dilution of physalis Peruviana Pulp, Brazilian Archieves of Biology and Technology. 56(03), 467-474, 2013.

3 BRASIL. Resolução RDC n²70, de 23 de set. de 2005 da Agência Nacional de Vigilância Sanitária do Ministério da Saúde. Aprova o Regulamento Técnico referente a Óleos Vegetais, Gorduras Vegetais e Creme Vegetal. Diário Oficial [da] República Federativa do Brasil, Poder Executivo, Brasília, DF, 23 set. 2005. Seção 1, n 184, p.372-3. Disponível em: www.anvisa.gov.br. Acesso em: 05 jun. 2015.

4 BYRNE, L.; BARKER, J.; PENNARUM-THOMAS, G.; DIAMOND, D. Digital imaging as a detector for generic analytical measurements. Trendas in Analitical Chemistry, v.19, n.8, 2000.

5 COSTA, G,B, FERNANDES, D,D,S., ALMEIDA, V,E., ARAUJO, T,S,P., MELO, J,P., DINIZ, P,H,G, Dias., VÉRAS, G., Digital image-based classification of biodiesel, Talanta, v.139, p.50-55, 2015.

6 DAVIES, E.R. The application of machine vision to food and agriculture: a review. The Imaging Science Journal, v.57, p.197-217, 2009.

7 DOMINGUEZ, M.A.; CENTURIÓN, M.E. Application of digital images to determine color in honey samples from Argentina, Microchemical Journal, V.118, p.110-114, Buenos Aires, 2014.

8 DOUGHERTY, E.R. Digital image processing methods. Marcel Dekker, New York, 1994.

9 FERNANDES, J.K. Aplicação de técnicas de análise de imagem para avaliação de parâmetros em alimentos. 77f. Dissertação (mestrado) - Universidade Federal do Paraná, Setor de Tecnologia, Programa de Pós-graduação em Tecnologia de Alimentos. Defesa: Curitiba, 2011.

10 FERNANDES, J.K.; UMEBARA, T.; LENZI, M.K.; ALVEZ, E.T.D. Image analysis for compôsition monitoring. commercial blends of olive and soybean oil. Acta Scientiarum-Technology. 35(02), 317-324, 2013

11 FERRARA, L.A; RAIMONDI, S. A.; d'EPISCOPO, L.; GUIDA. L; RUSSO, D. A.; TEODORO, M. Olive oil and reduced need for antihypertensive medications. Arch Intern Med. v.160, p.837-842, 2000. 
FILHO, O. M.; NETO, H.V. Processamento Digital de Imagens. Rio de Janeiro: Brasport, 1999.

13 GONILELLI, C. G.; SANTOS. M. S.; MARCONCIN, L. V.; COLNAGO, L. A. Uso da RMN-DT no desenvolvimento e validação de método para detecção de adulteração em azeites de oliva comerciais lacrados. Anais da V Jornada Cientifica -Embrapa São Carlos, São Carlos, 2013

HARVEY, D. Modern Analytical Chemistry. $1^{\circ} \mathrm{ed}$. DePauw University: McGraw-Hill, 2007.

16 ISFER, L. A. D; LENZI, M. K.; LENZI, E. K. Identification of biochemical reactors using fractional differential equations, Latin American Applied Research, v. 40, p.193-198, 2010.

17 KOYA, T. K. Sistema Automático para Contagem das Células do Sangue. (Dissertação de mestrado). Universidade Federal do Rio Grande do Sul - UFRGS, Porto Alegre, 2001

18 LEVENBERG, K. A method for the solution of certain problems in least squares. Quarterly of Applied Mathematics, v.2, p.164-168, 1944.

19 MARQUARDT, D. An algorithm for least-squares estimation of nonlinear parameters. SIAM Journal on Applied Mathematics, v.11, p.431-441, 1963.

20 MASAWA, T. P.; HARFIELD, A., NAMWONG, A. An iPhone-based digital image colorimeter for detecting tetracycline in milk, Food Chemistry, V. 184, p.23-29, Phitsanulok, 2015.

21 MACHADO, A. M. L. Extração automática de contornos de edificações utilizando imagem gerada por câmara digital de pequeno formato e dados lidar. 213f. Tese (Doutorado em Ciências Geodésicas) - Setor de ciências da Terra, Universidade Federal do Paraná, Curitiba, 2006.

22 MILANEZ, M. T. D. K.; PONTES, C. J. M. Classification of edible vegetable oil using digital image and pattern recognition techniques. Microchemical Journal, v.113, p.10-16, 2013.

23 RUSS, J. C. The image processing handbook, CRC Press, Boca Raton, 1995. SKOOG, D. A.; WEST, D. M; HOLLER, F. J. Fundamentals of analytical chemistry. 9th . ed. Brooks Cole, 2013. 